\title{
A case of acute myelogenous leukemia characterized by arterial and venous thrombosis
}

\author{
Huasheng Liang ${ }^{1}$, Mingchuan $\mathrm{Ba}^{1}$, Chen $\mathrm{Li}^{1}$, Haoping $\mathrm{Li}^{1}$, Zhiqiang Guo ${ }^{1}$, Pengcheng He ${ }^{1,2}$, Chunying Lin ${ }^{1}$ \\ ${ }^{1}$ Department of Cardiology, Guangdong Provincial People's Hospital's Nanhai Hospital, Foshan, China; ${ }^{2}$ Department of Cardiology, Guangdong \\ Cardiovascular Institute, Guangdong Provincial People's Hospital, Guangdong Academy of Medical Sciences, Guangzhou, China \\ Correspondence to: Chunying Lin, MD. Department of Cardiology, Guangdong Provincial People’s Hospital's Nanhai Hospital, Foshan 528251, \\ China. Email: chunyinglin@163.com.
}

\begin{abstract}
Acute myelogenous leukemia (AML) is a malignant disease of the hematopoietic system, characterized by features of bone marrow insufficiency and organ infiltration by leukemic cells. Venous thrombosis in AML patients is uncommon, compared to bleeding; therefore in patients with AML, simultaneous occurrence of venous and arterial thrombosis is a rather rare presentation. We reported an unusual case of anti-phospholipid antibody syndrome secondary to AML characterized by venous and arterial thrombosis. A 70-year-old man with deep venous thrombosis (DVT) of the left leg confirmed by Doppler was seen in our clinic. During treatment with a Vitamin K antagonist (3 $\mathrm{mg}$ daily of Warfarin) and a low molecular weight heparin (LMWH), he developed an acute pulmonary embolism and an acute inferior wall ST elevation myocardial infarction (STEMI), a result of right coronary artery embolism. His full blood count showed leukocytosis and thrombocytopenia. Lupus anticoagulant and anti-cardiolipin antibodies were positive. A bone marrow aspirate test showed results consistent with AML (FAB class M1). A diagnosis of antiphospholipid antibody syndrome secondary to AML characterized by coronary artery embolism, pulmonary embolism and left leg DVT was eventually established. He received anticoagulation with a low dose of warfarin after refusing chemotherapy. He however died of cerebral hemorrhage despite the fact that the INR was in the normal therapeutic range. It is challenging to anticoagulated AML patients complicated by multiple vascular thromboses and thrombocytopenia.
\end{abstract}

Keywords: Acute myelogenous leukemia (AML); deep venous thrombosis (DVT); pulmonary embolism; acute myocardial infarction; antiphospholipid antibody syndrome

Submitted May 30, 2020. Accepted for publication Aug 14, 2020.

doi: $10.21037 / \mathrm{cdt}-20-486$

View this article at: http://dx.doi.org/10.21037/cdt-20-486

\section{Introduction}

Acute myelogenous leukemia (AML) is a malignant disease of the hematopoietic system, characterized by features of bone marrow insufficiency such as anemia and thrombocytopenia; and also by granulocytosis which is a result of proliferation of these abnormal hematopoietic cells of the white blood cell line. There is also organ infiltration by these abnormal or malignant hematopoietic cells. Most patients with AML present with anemia, infections and bleeding as a result. Venous thrombosis in AML is less common, compared to bleeding; therefore in patients with AML, venous and arterial thrombosis is a rare presentation (1). We encountered an unusual case of anti-phospholipid antibody syndrome secondary to AML characterized by DVT, pulmonary embolism and acute myocardial infarction caused by right coronary artery embolism. The characteristics and possible reasons for arterial and venous thrombosis in patients with AML are discussed in this report. We present the following article in accordance with the CARE reporting checklist (available at http://dx.doi. org/10.21037/cdt-20-486). 

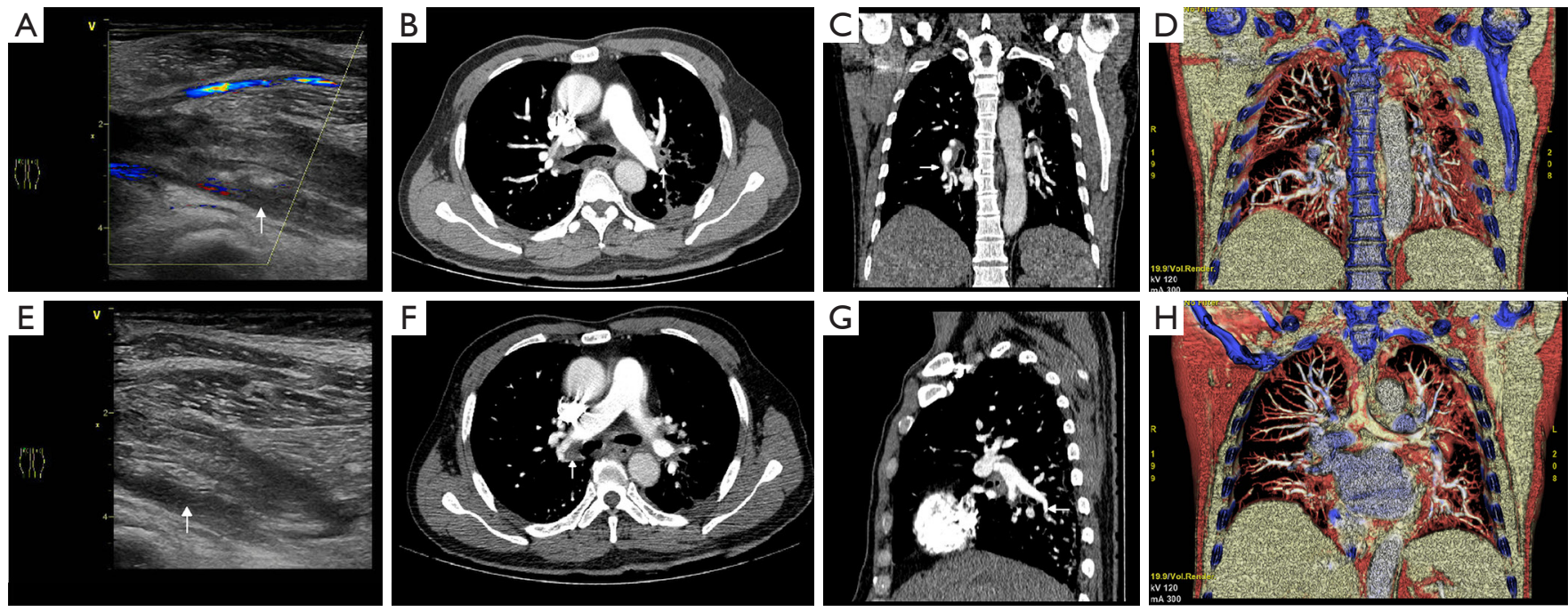

Figure 1 The color Doppler ultrasound of left leg (A,B) showed non-compressible popliteal and peroneal veins with abnormal echo (arrows). The CPTA (C,D,E,F) and three-dimensional reconstruction $(\mathrm{G}, \mathrm{H})$ showed emboli (arrows) in the terminal branches of both pulmonary arteries $(\mathrm{C}, \mathrm{D})$ and the branches of both lower pulmonary arteries $(\mathrm{E}, \mathrm{F})$.

\section{Case presentation}

A 70-year-old man presented to the general surgery department of our hospital with a 2 -week history of a painful progressive left calf swelling without associated fever or chills. He had no personal or family history of venous thromboembolism or coronary artery disease. He had no history of diabetes mellitus, hypertension or dyslipidemia. He had however been treated successfully in the past for pulmonary tuberculosis with no residual sequelae. He drank no alcohol and denied smoking or recreational drug use.

On examination, he was an elderly man in pain with an obvious swollen left leg. He was afebrile and had no respiratory distress. He was not tachycardic and had normal blood pressure. His entire left leg was swollen and tender with a calf circumference of $41 \mathrm{~cm}$ (measured $10 \mathrm{~cm}$ below the tibial tuberosity), whiles the right leg measured $35.5 \mathrm{~cm}$ and was neither swollen nor tender. The rest of the physical examination was unremarkable.

Lab results showed a very mild normocytic anemia and mild leukocytosis. He had severe thrombocytopenia and a severely elevated C-reactive protein (almost 20 times the upper border of normal). He also had significant elevated D-dimer (over 40 times the upper border of normal). A color Doppler ultrasound of his left leg showed noncompressible popliteal and peroneal veins, indicating a left leg DVT (Figure 1).

He was diagnosed with DVT of the left leg, admitted and started on a dual therapy of Low molecular weight heparin (LMWH) (Nadoparin calcium $0.4 \mathrm{~mL}$ ) and a Vitamin $\mathrm{K}$ antagonist (Warfarin $3 \mathrm{mg}$ ). The LMWH was stopped the following day on account of the thrombocytopenia, and patient was transfused with one unit of cell-matched platelet concentrate.

On the third day of admission, the patient complained of a sudden onset severe chest pain and difficulty breathing without hemoptysis. His arterial blood gases on room air were normal. An urgent CT pulmonary angiogram (CTPA) was ordered, which showed emboli in the terminal branches of both pulmonary arteries and the branches of both lower pulmonary arteries (Figure 1). A diagnosis of acute pulmonary embolism was made and the patient was transferred to the intensive care unit (ICU).

He was continued on Warfarin $3 \mathrm{mg}$ and Nadoparin $0.4 \mathrm{~mL} / \mathrm{q} 12 \mathrm{~h}$ was re-started. Patient's International Normalized Ratio (INR) was found to be progressively rising till they became markedly elevated (over 5 times the upper border of normal after 6 days of dual anticoagulation therapy). Both warfarin and the LMWH were stopped and the patient was transfused with $600 \mathrm{mLs}$ of fresh frozen plasma.

In spite of the above therapy, the retrosternal chest pain persisted over the following two days, without radiation to the neck, jaws or left arm. Cardiac enzymes and a 12lead ECG were ordered which showed markedly elevated cardiac enzymes and Troponin (over 19 times above the 

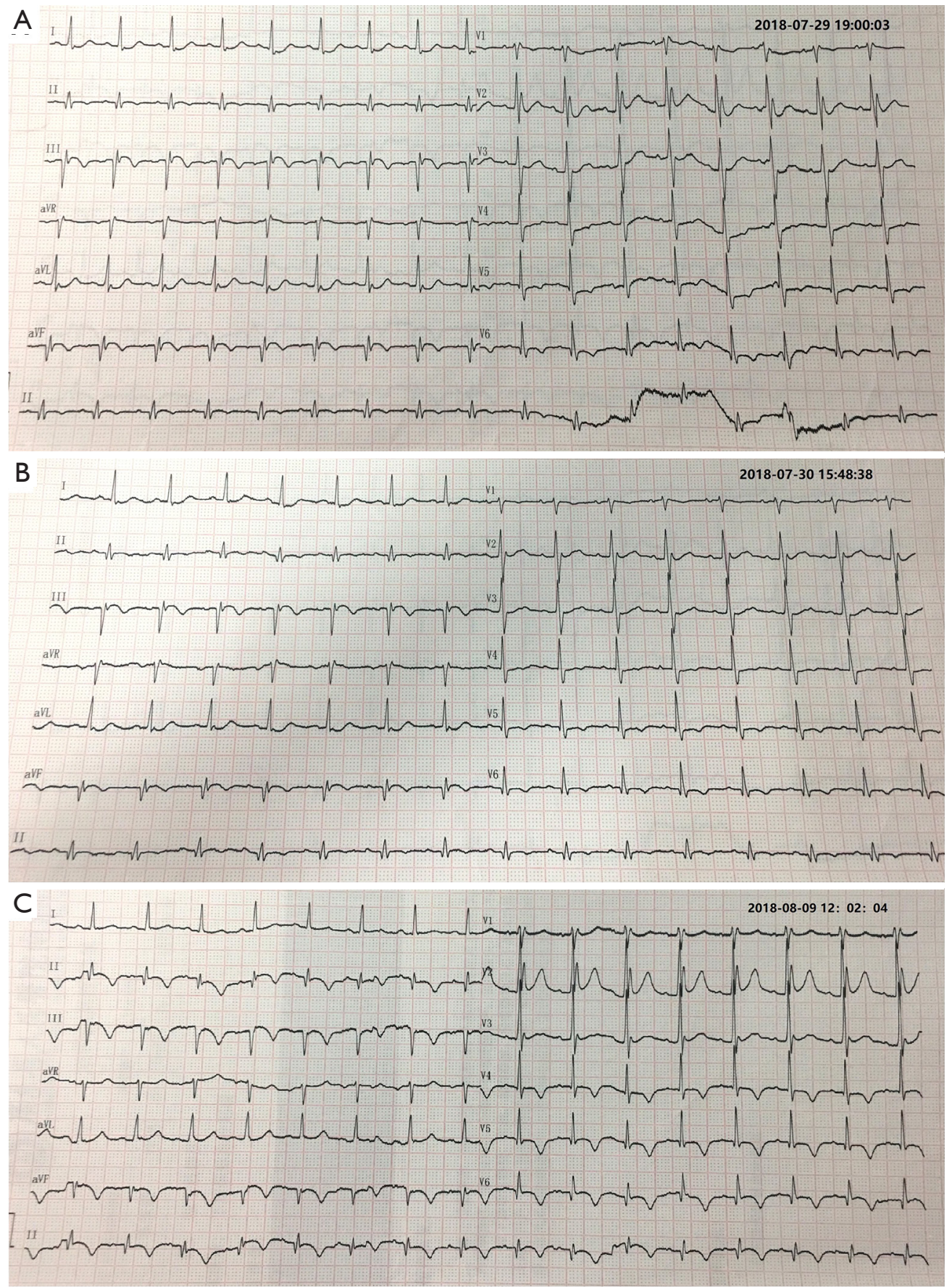

Figure 2 The 12-lead ECG (A,B,C) showed an acute inferior wall STEMI and dynamic change of ST segment elevation in leads II, III, and avF, during hospitalization.

upper border of normal) and an acute inferior wall STEMI (Figure 2A).

On consultation with the cardiologists, a diagnosis of acute inferior wall STEMI was confirmed and an emergent
Coronary Angiography with PCI was recommended. The patient and his relatives however refused to give consent and opted for conservative management. Follow up ECGs showed dynamic ST segment changes (Figure $2 A, B, C$ ) 
A
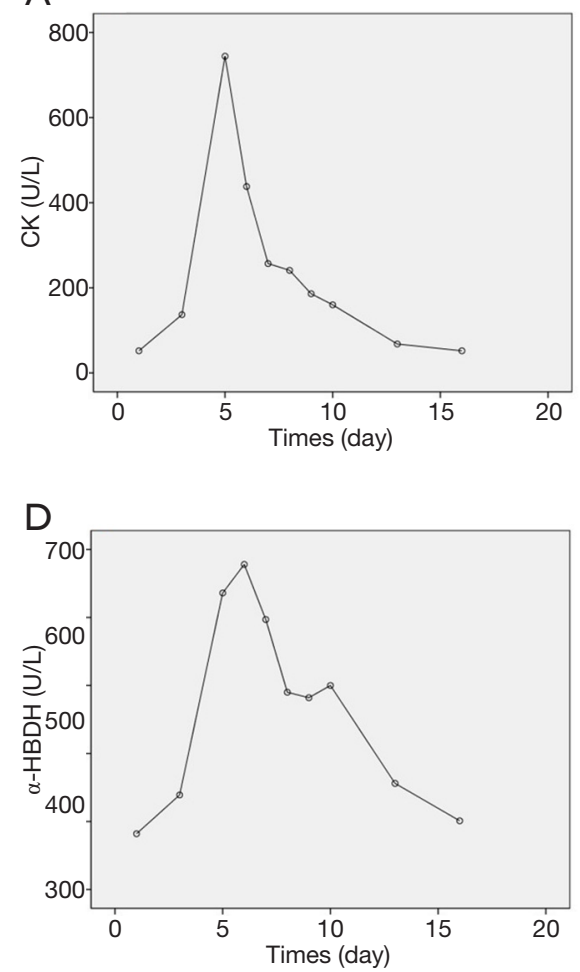

B

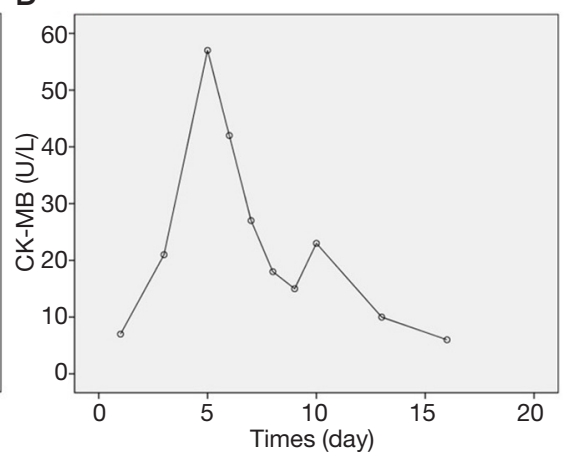

E

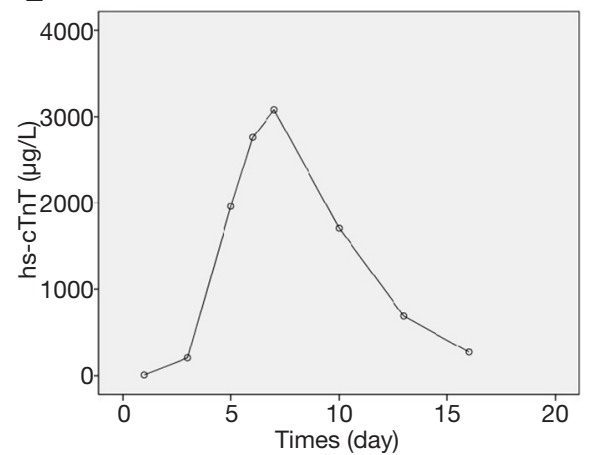

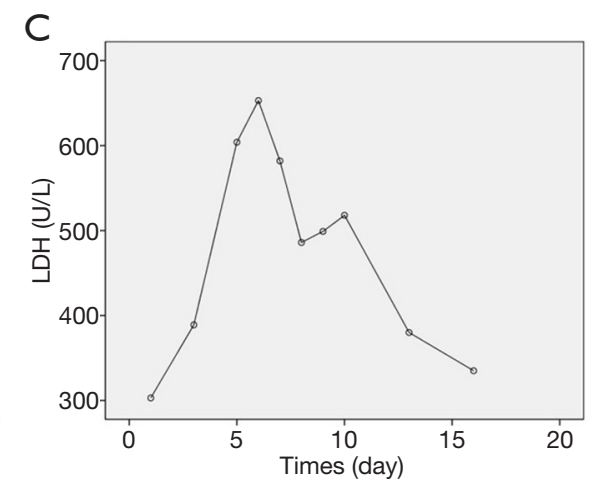

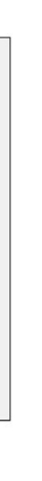

Figure 3 The dynamic change of myocardial enzyme (A: CK, B: CK-MB, C: LDH, D: a-HBDH, E: hs-cTnT) during hospitalization. $\mathrm{LDH}$, lactate dehydrogenase; a-HBDH, alpha-hydroxybutyric dehydrogenase.

further confirming the diagnosis of acute inferior wall STEMI.

He was managed on Clopidogrel $75 \mathrm{mg}$ daily and Warfarin was reduced to $1.5 \mathrm{mg}$ nocte, without Aspirin, on account of the low platelet count. The low platelet count however persisted so clopidogrel had to be stopped and patient was left only on warfarin. After two weeks of admission at the ICU, he became stable and was transferred to the cardiology ward for further management.

Repeat labs showed that cardiac enzymes and troponin had fallen to baseline levels (Figure 3) and the platelet count had moderately improved (from $39 \times 10^{9} / \mathrm{L}$ on the day of admission to $61 \times 10^{9} / \mathrm{L}$ after transfusion with a unit of platelet concentrate; normal range: $100 \times 10^{9}-300 \times 10^{9} / \mathrm{L}$ ).

Further investigations revealed a positive anti-cardiolipin and lupus anticoagulant antibodies. An autoantibody screen; including ANA, anti-dsDNA, rheumatoid factor (RF), and anti-neutrophil cytoplasmic antibody (ANCA), were however negative. Echocardiography showed regional wall motion abnormality (inferior wall) with a residual left ventricular systolic dysfunction (Left ventricular ejection fraction of $41 \%$ ), as well as a mild mitral and tricuspid regurgitation.

Coronary artery CTA showed complete occlusion of proximal RCA without any obvious stenosis or atherosclerosis in the left coronary artery or any of its branches (Figure 4). A bone marrow aspirate cytology and biopsy was done in his fourth week of admission which showed a predominance of large primitive cells with $92 \%$ granulocytes in the bone marrow smear and 29\% myeloblasts in the peripheral blood smear, consistent with AML (FAB M1) (Figure 5).

A final diagnosis of antiphospholipid antibody syndrome (APS) secondary to AML (FAB M1) complicated by Acute myocardial infarction (AMI), Pulmonary embolism (PE) and Deep venous thrombosis (DVT) was made. Induction chemotherapy for AML was recommended after consultation with the hematologists but was declined by the patient and his relatives. After discharge, patient continued to refuse to undergo chemotherapy in other hospitals despite being urged to do so by his relatives.

A month after discharge, patient presented to our emergency department in a deep coma. An urgent head CT scan was done which showed a massive right intracerebral 

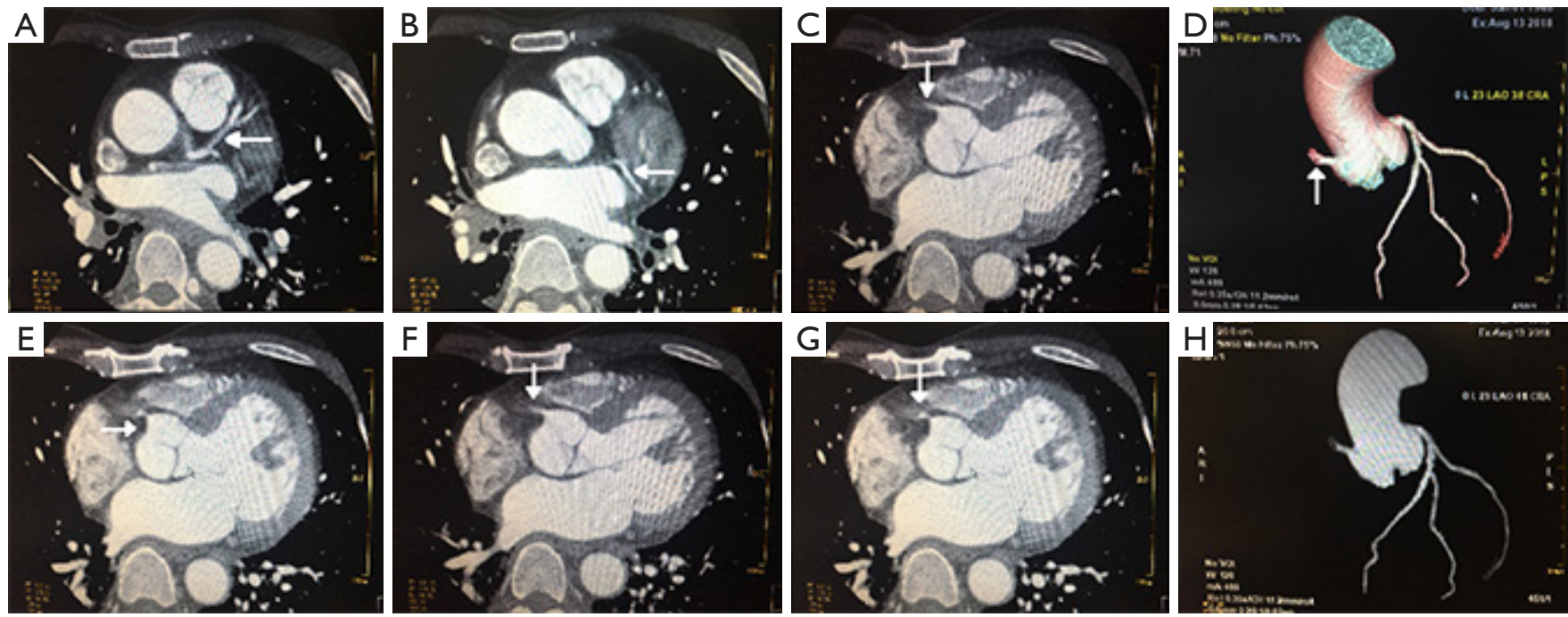

Figure 4 The coronary artery CTA (A-F) and three-dimensional reconstruction $(\mathrm{G}, \mathrm{H})$ showed complete occlusion (arrows) of initial segment of the right coronary artery $(\mathrm{C}-\mathrm{H})$, without any atherosclerosis or stenosis in the left coronary artery and its branches $(\mathrm{A}, \mathrm{B})$.
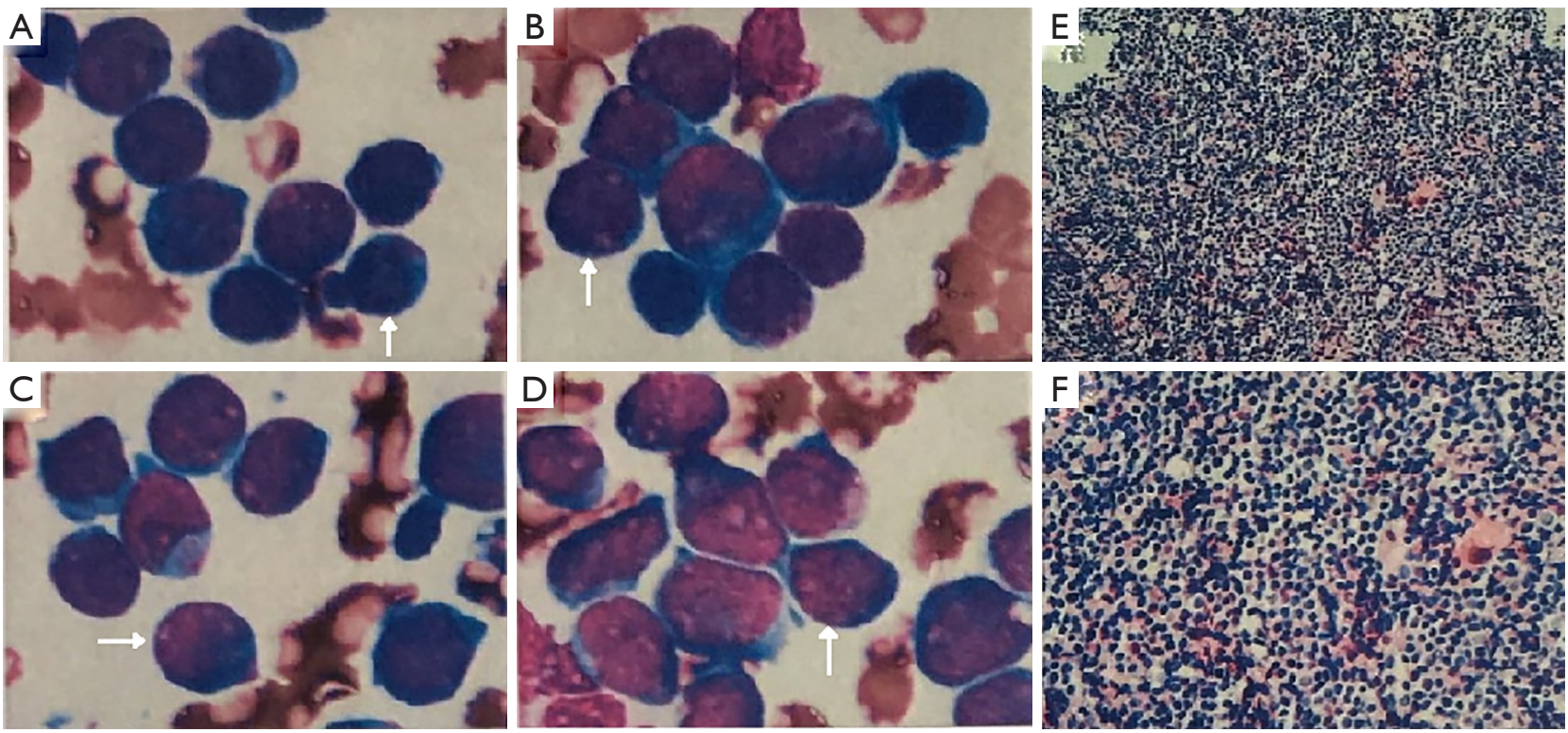

Figure 5 The bone marrow aspiration with Wright stain (A-D, 100x) and biopsy with HE stain (E,F, 20x) showed acute myelogenous leukemia (FAB, M1) with a predominance of large primitive cells (arrows).

hemorrhage, a subarachnoid hemorrhage and brain herniation (Figure 6). He unfortunately died shortly after presentation from the brain herniation.

All procedures performed in studies involving human participants were in accordance with the ethical standards of the institutional and/or national research committee(s) and with the Helsinki Declaration (as revised in 2013). Written informed consent was obtained from the patient and his relatives.

\section{Discussion}

This is a case report of an unusual case of acute myelogenous 

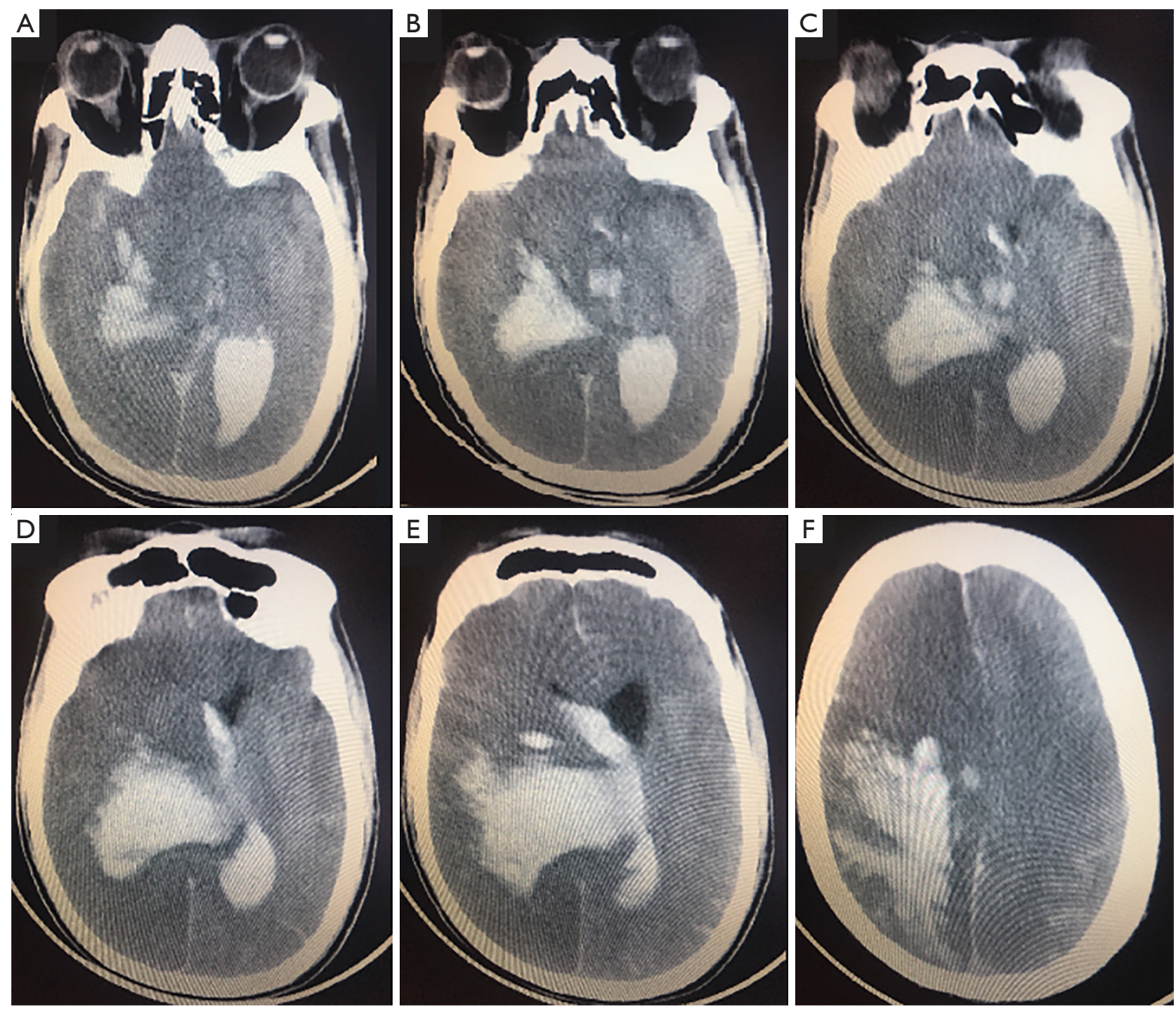

Figure 6 The cranial CT scan showed cerebral hemorrhage, subarachnoid hemorrhage, as well as herniation of brain.

leukemia (AML) presenting as Antiphospholipid antibody syndrome (APS). The initial diagnosis of APS was based on the presence of multiple vascular thromboses including a left leg DVT, PE and an AMI secondary to right coronary artery embolism; with accompanying thrombocytopenia and positive antiphospholipid antibodies.

AML commonly presents with anemia, hemorrhage, recurrent infections and organomegaly resulting from organ infiltration with leukemic cells. Hemorrhagic or thrombotic events may occur in patients with AML, but thrombotic events are less common because of thrombocytopenia with its accompanying coagulopathy in these patients (1). Major thrombotic events may commonly occur in AML patients with hyperleukocytosis (WBC $>10^{11} / \mathrm{L}$ ), especially for acute promyelocytic leukemia (APL) $(2,3)$. In a review of APL-related thrombosis, DVT/PE was the most common thrombotic event, followed by cardiovascular events (mainly coronary artery thrombosis) and cerebrovascular events(stroke or Dural Sinus thrombosis) $(1,4)$. Although some previous studies have reported on some AML patients presenting with either DVT, PE or AMI occurring alone (4-10); both arterial and venous thromboembolic events characterized by all three occurring together as in this case is uncommon in AML patients.

The association between AML and thrombosis is well documented; however the pathogenesis of thrombosis 

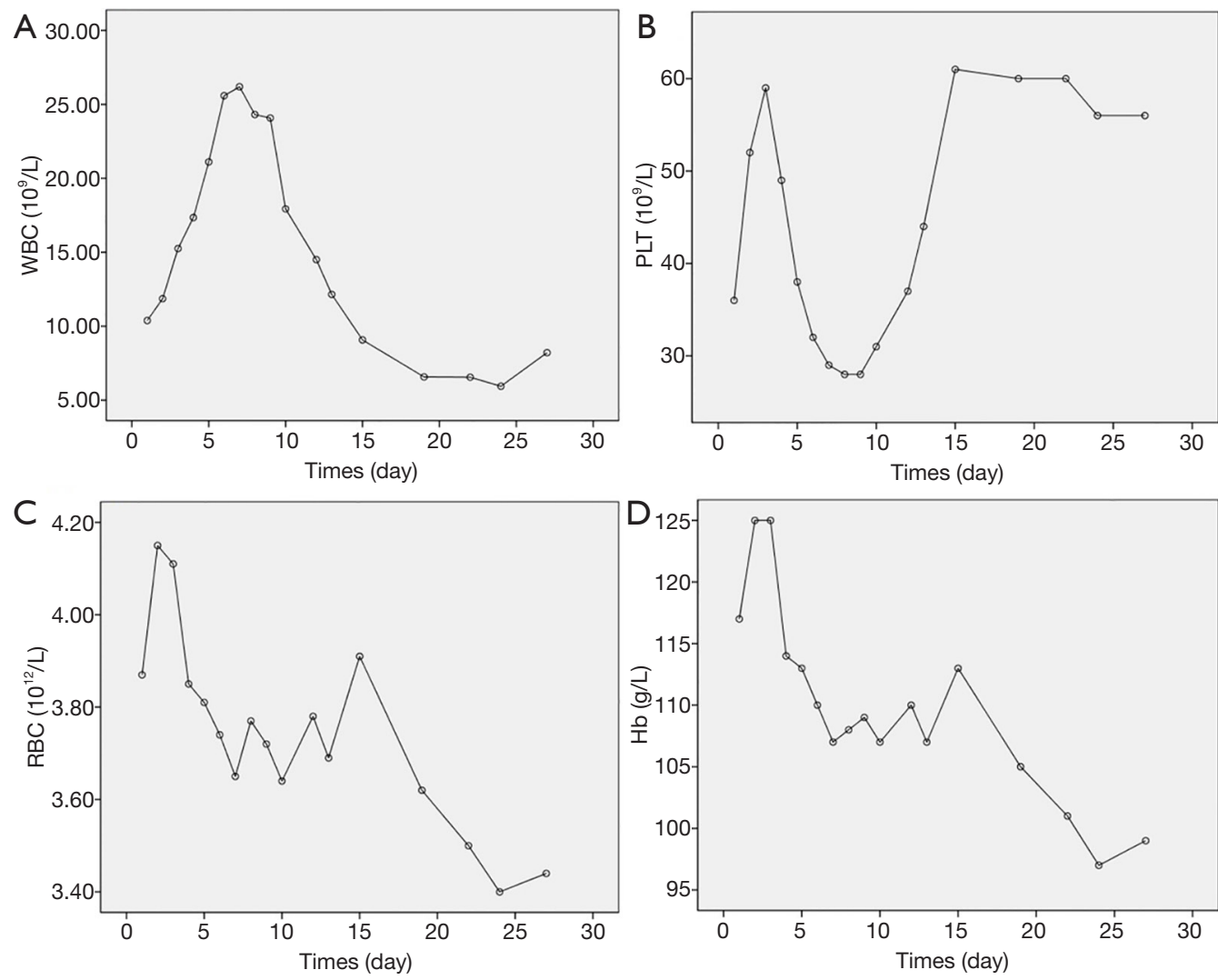

Figure 7 The dynamic change of blood cell count (A: WBC; B: PLT; C: RBC; D: Hb) during hospitalization. PLT, platelet; RBC, red blood cell; Hb, hemoglobin.

in AML remains unclear. Several hypotheses have been postulated, such as production of prothrombotic cytokines by the abnormal leukemic cells, direct expression of tissue factor and cancer procoagulant by these cells, disseminated intravascular coagulation, infiltration of blood vessels by leukemic cells, occlusion of the blood vessel by a leukemic thrombus, leukostasis syndrome and deficiency of coagulation factors $(10,11)$.

Because of their smaller luminal diameter, the microvasculature is most susceptible to leukemic cell infiltration, usually affecting the central nervous system and the respiratory system, while major arterial thromboembolic events are uncommon, especially in epicardial coronary arteries due to their larger caliber (8). Why this patient suffered from such multiple vascular thromboses remains a dilemma.

It is worthy of note, that the highest WBC count for this patient during admission was only $26.19 \times 10^{9}$ (Figure 7) which makes leukostasis syndrome less likely. In addition, the coagulation test showed prolonged prothrombin time and activated partial thromboplastin time, without reduction of fibrinogen. There was also no evidence of bleeding, which indicated that disseminated intravascular coagulation was not likely either.

The fact that the patient presented with multiple vascular thrombosis and thrombocytopenia with a positive anticardiolipin and lupus anticoagulant led to the diagnosis of APS secondary to AML which is considered as the aetiology of the venous and arterial thrombosis in this patient.

It is well known that APS is a hypercoagulable condition characterized by venous or arterial thrombosis, or both, in the presence of antiphospholipid antibodies. APS can be primary, occurring in patients without concomitant disease, or secondary to conditions such as systemic lupus erythematous, infections, drug use, or malignancies. Several studies have reported the presence of APS in acute lymphoblastic leukemia and acute myelogenous leukemia; however, the pathogenesis of APS in AML remains unclear. It is hypothesized that production of prothrombogenic cytokines by abnormal leukemic cell may lead to immune 
dysregulation and autoantibody formation, thereby activating the coagulation cascade, eventually leading to multiple vascular thrombosis $(12,13)$.

In the treatment of AML patients with arterial and venous thrombosis, both anticoagulant and antiplatelet therapy are necessary to reduce new thrombotic events. However, there was a high risk of bleeding in this patient, because of the thrombocytopenia. In addition, he also had anaemia. This patient was initially started on anticoagulation with warfarin $1 \mathrm{mg} / \mathrm{qn}$ and a single antiplatelet (clopidogrel, $75 \mathrm{mg} / \mathrm{qd}$ ) therapy during hospitalization, but antiplatelet therapy with clopidogrel $(75 \mathrm{mg} / \mathrm{qd})$ was stopped, due to recurrent positive faecal occult blood test results. Finally, only anticoagulation therapy with warfarin $(1 \mathrm{mg} / \mathrm{qn})$ was continued to maintain an international normalized ratio (INR) between 2 and 3. Thus anticoagulation therapy was preferred over antiplatelet therapy in this patient.

Upon discharge, the patient continued anticoagulation with warfarin $(1 \mathrm{mg} / \mathrm{qn})$, and the INR was among normal therapeutic window (2.0 to 3.0), after one month follow up. He still eventually died of intracerebral hemorrhage and brain herniation. It can be inferred from this case that it is very important to monitor patients on anticoagulant therapy for AML complicated by venous thrombosis and thrombocytopenia very closely post discharge. As to whether it is safer to use NOACs for such patients remains uncertain. Lastly, in relation to the etiology, it was necessary for the patient to have had induction chemotherapy for AML. However, the patient refused chemotherapy.

\section{Conclusions}

In conclusion, the above is a case report of a rare case of antiphospholipid antibody syndrome secondary to AML in a patient presenting with multiple arterial and venous thrombosis, including a DVT of the left leg, PE and AMI caused by right coronary artery thromboembolism. It sums up the need to estimate the risk of bleeding, when anticoagulation therapy is being considered for AML patients with multiple vascular thrombosis and thrombocytopenia.

\section{Acknowledgments}

We wish to appreciate the following for their help in the translation of our report to professional English: Kyerewah Akosua, Bobbie Sarfo (a cardiologist from Korle-Bu Teaching Hospital, The Republic of Ghana) and Abdul-
Subulr Yakubu (a cardiologist from Tamale Teaching Hospital, The Republic of Ghana).

Funding: None.

\section{Footnote}

Reporting Checklist: The authors present the study in accordance with the CARE reporting checklist. Available at http://dx.doi.org/10.21037/cdt-20-486

Peer Review File: Available at http://dx.doi.org/10.21037/ cdt-20-486

Conflicts of Interest: All authors have completed the ICMJE uniform disclosure form (available at http://dx.doi. org/10.21037/cdt-20-486). The authors have no conflicts of interest to declare.

Ethical Statement: The authors are accountable for all aspects of the work in ensuring that questions related to the accuracy or integrity of any part of the work are appropriately investigated and resolved. All procedures performed in studies involving human participants were in accordance with the ethical standards of the institutional and/or national research committee(s) and with the Helsinki Declaration (as revised in 2013). Written informed consent was obtained from the patient and his relatives.

Open Access Statement: This is an Open Access article distributed in accordance with the Creative Commons Attribution-NonCommercial-NoDerivs 4.0 International License (CC BY-NC-ND 4.0), which permits the noncommercial replication and distribution of the article with the strict proviso that no changes or edits are made and the original work is properly cited (including links to both the formal publication through the relevant DOI and the license). See: https://creativecommons.org/licenses/by-nc-nd/4.0/.

\section{References}

1. Ziegler S, Sperr WR, Knöbl P, et.al. Symptomatic venous thromboembolism in acute leukemia. Incidence, risk factors, and impact on prognosis. Thromb Res 2005;115:59-64.

2. Rashidi A, Silverberg ML, Conkling PR, et al. Thrombosis in acute promyelocytic leukemia. Thromb Res 2013;131:281-9.

3. Chang H, Kuo MC, Shih LY, et al. Acute Promyelocytic 
Leukemia-Associated Thrombosis. Acta Haematol 2013;130:1-6.

4. Kutiyal AS, Dharmshaktu P, Kataria B, et al. A Rare Occurrence of Simultaneous Venous and Arterial Thromboembolic Events - Lower Limb Deep Venous Thrombosis and Pulmonary Thromboembolism as Initial Presentation in Acute Promyelocytic Leukemia. Clin Med Insights Oncol 2016;10:1-4.

5. Skalidis E, Anastasiou I, Konstantinou I, et al. Leukemic Blast Clot Causing ST-Segment Elevation Myocardial Infarction. JACC Cardiovasc Interv 2018;11:1656-7.

6. Özkurt ZN, Aypar E, Sarifakiogullari S, et al. Acute myocardial infarction as a finding of acute promyelocytic leukemia-related coagulation disorder. Blood Coagul Fibrinolysis 2015;26:949-52.

7. Jao GT, Knovich MA, Savage RW, et al. ST-Elevation Myocardial Infarction and Myelodysplastic Syndrome with Acute Myeloid Leukemia Transformation. Tex Heart Inst J 2014;41:234-7.

8. Muñiz AE. Myocardial Infarction and Stroke as the Presenting Symptoms of Acute Myeloid Leukemia. J

Cite this article as: Liang $\mathrm{H}, \mathrm{Ba} \mathrm{M}, \mathrm{Li} \mathrm{C}, \mathrm{Li} \mathrm{H}$, Guo Z, He $\mathrm{P}$, Lin C. A case of acute myelogenous leukemia characterized by arterial and venous thrombosis. Cardiovasc Diagn Ther 2020;10(5):1332-1340. doi: 10.21037/cdt-20-486
Emerg Med 2012;42:651-4.

9. Cahill TJ, Chowdhury O, Myerson SG, et al. Myocardial Infarction with Intracardiac Thrombosis as the Presentation of Acute Promyelocytic Leukemia. Circulation 2011;123:e370-2.

10. Chang H, Lin TL, Ho WJ, et al. Acute Myeloid Leukemia Associated with Acute Myocardial Infarction and Dural Sinus Thrombosis: The Possible Role of Leukemia-related Hyperhomocysteinemia. J Chin Med Assoc 2008;71:416-20.

11. Del Principe MI, Del Principe D, Venditti A. Thrombosis in adult patients with acute leukemia. Curr Opin Oncol 2017;29:448-54.

12. Orsino A, Schneider R, DeVeber G, et al. Childhood Acute Myelomonocytic Leukemia (AML-M4) Presenting as Catastrophic Antiphospholipid Antibody Syndrome. J Pediatr Hematol Oncol 2004;26:327-30.

13. Saxena SK, Bin Salih SA, Al-Jizeeri AH, et al. Acute myeloblastic leukemia in a patient with primary antiphospholipid syndrome. Saudi Med J 2003;24:1013-5. 